\title{
The fundamental parameters of the Ap star 78 Virginis
}

\section{Could 78 Vir be a rapidly oscillating Ap star?`}

\author{
K. Perraut ${ }^{1,2}$, M. Cunha ${ }^{3}$, I. Brandão ${ }^{3}$, J. Loridat ${ }^{1,2}$, D. Mourard ${ }^{4}$, A. Meilland ${ }^{4}$, N. Nardetto ${ }^{4}$, H. McAlister ${ }^{5,6}$ \\ T. A. ten Brummelaar ${ }^{6}$, J. Sturmann ${ }^{6}$, L. Sturmann ${ }^{6}$, N. Turner ${ }^{6}$, C. Farrington ${ }^{6}$, and N. Vargas ${ }^{6}$ \\ 1 Univ. Grenoble Alpes, IPAG, 38000 Grenoble, France \\ e-mail: karine.perraut@obs.ujf-grenoble.fr \\ 2 CNRS, IPAG, 38000 Grenoble, France \\ 3 Instituto de Astrofísica e Ciências do Espaço, Universidade do Porto, CAUP, Rua das Estrelas, 4150-762 Porto, Portugal \\ 4 Laboratoire Lagrange, UNS-CNRS-OCA, CS 34229, 06304 Nice Cedex 4, France \\ 5 Georgia State University, PO Box 3969, Atlanta GA 30302-3969, USA \\ ${ }^{6}$ CHARA Array, Mount Wilson Observatory, 91023 Mount Wilson CA, USA
}

Received 23 March 2015 / Accepted 13 May 2015

\begin{abstract}
Context. Determining the effective temperature of Ap stars, including the roAp stellar pulsators, is a difficult task owing to their strong magnetic field and their related spotted surfaces. It is, however, an important step towards constraining models of their complex atmosphere and testing proposed pulsation excitation mechanisms.

Aims. Using the unique angular resolution provided by long-baseline visible interferometry, we aim at deriving accurate angular diameters of a number of Ap targets, so as to determine their unbiased effective temperature $\left(T_{\text {eff }}\right)$ and their accurate position in the Hertzsprung-Russell diagram, to estimate their mass and age, and to test non-adiabatic pulsation models. Interferometric results on four Ap stars have been published in earlier works. Here we report the results on a fifth, significantly hotter star.

Methods. We observed 78 Vir with the visible spectrograph VEGA installed at the combined focus of the CHARA long-baseline optical array. We derived the limb-darkened diameter of this Ap star from our interferometric measurements. Based on photometric and spectroscopic data available in the literature, we estimated the star's bolometric flux and used it, in combination with its parallax and angular diameter, to determine the star's luminosity and effective temperature. We then used the derived fundamental parameters to perform a non-adiabatic pulsation analysis.

Results. We determined a limb-darkened angular diameter of $0.346 \pm 0.006$ mas and deduced a linear radius of $R=2.11 \pm 0.04 R_{\odot}$. Considering a bolometric flux of $2.73 \pm 0.2010^{-7} \mathrm{erg} / \mathrm{cm}^{2} / \mathrm{s}$ we obtained a luminosity of $L / L_{\odot}=27 \pm 2$ and an effective temperature of $T_{\mathrm{eff}}=9100 \pm 190 \mathrm{~K}$. The non-adiabatic pulsation modeling allows us to predict that high overtone pulsations could be excited in 78 Vir at frequencies ranging from 1.2 to $1.9 \mathrm{mHz}$, provided that the magnetic field is capable of suppressing envelope convection in the polar regions.

Conclusions. Visible long-baseline interferometry is a unique means of deriving accurate fundamental parameters of Ap stars. The Ap star 78 Vir is found to be a promising roAp-star candidate and one that would allow us to extend recent tests on the roAp stars' excitation mechanism towards the blue edge of the instability strip. Asteroseismic data of this star would, therefore, be of strong interest.
\end{abstract}

Key words. methods: observational - techniques: high angular resolution - techniques: interferometric - stars: individual: 78 Vir stars: fundamental parameters

\section{Introduction}

78 Virginis (78 Vir; HD 118022; HR 5105; $\left.m_{V}=4.94\right)$ is a bright Ap star classified as A1pCrSrEu by Cowley et al. (1969). It is a member of the magnetic chemically peculiar (CP) stars, usually called Ap stars. They are cool magnetic stars, ranging in spectral type from F0 to A3, with corresponding effective temperatures between 7000 and $10000 \mathrm{~K}$ (Kurtz \& Martinez 2000). They have strong and often variable lines of $\mathrm{Sr}$, and $\mathrm{Cr}$ and other iron peak elements. Some of the coolest stars display non-radial pulsations in high overtone acoustic modes with periods between 6 and 23 min (Kurtz et al. 2006; Alentiev et al. 2012) and are named rapidly oscillating Ap stars (roAp stars).

\footnotetext{
* Based on observations made with the VEGA/CHARA spectro-interferometer.
}

$78 \mathrm{Vir}$ is the first star other than the sun in which a magnetic field was discovered by Babcock (1947). The surface magnetic field configuration was reconstructed by Khalack \& Wade (2006): a slightly decentered magnetic dipole with a polar surface intensity of about $3.3 \mathrm{kG}$ describes correctly the global magnetic field structure.

The effective temperature of 78 Vir was derived by Monier (1992) using the InfraRed Flux Method (IRFM, Blackwell \& Shallis 1977) and the fit of the energy distribution. Both methods lead to an effective temperature of $9200 \pm 200 \mathrm{~K}$, which is much lower than the previous determinations ranging from $9500 \mathrm{~K}$ up to $10700 \mathrm{~K}$ (Jugaku \& Sargent 1968). This lower effective temperature places 78 Vir close to the blue edge of the theoretical instability strip for roAp stars (Cunha 2002), making it a potential test-case for constraining non-adiabatic pulsation models, as recently illustrated by Cunha et al. (2013). 
However, that potential can only be realized if the effective temperature and luminosity of the star are known with significant accuracy. As a matter of fact, determining the effective temperature of peculiar stars is an important but very difficult task, particularly because of the complexity of their atmosphere. In Ap stars, the strong magnetic field is supposed to play a central role in the abundance distribution by stabilizing the external layers, by partially or fully suppressing the convection, and by contributing to microscopic diffusion. Such vertical and horizontal microscopic diffusion of the chemical elements (Michaud 1970; Stift \& Alecian 2012) might lead to spotted surfaces that have been reconstructed by Doppler or Zeeman-Doppler imaging for several Ap stars (Lüftinger et al. 2003; Kochukhov et al. 2004; Lüftinger et al. 2010; Kochukhov \& Wade 2010). Because of these abnormal surface layers, atmosphere models of Ap stars are extremely complex and the consequent determination of effective temperature (hence radius, assuming one knows the luminosity) by spectroscopic data is subject to difficulties. Determining accurate fundamental parameters by a method as independent as possible of an atmosphere model is thus required for performing asteroseismic investigations of these stars (Cunha et al. 2003, 2007), including the testing of non-adiabatic pulsation models, and it is also an efficient way to improve the modeling of their atmosphere. Modern long-baseline interferometry, working in the visible range like VEGA on the CHARA array (Mourard et al. 2009), reaches an angular resolution as low as 0.2 milliseconds of arc (mas), which allows several Ap stars to be resolved despite their very small angular size (i.e. $\leq 1$ mas).

In this paper, we report our interferometric observations of 78 Vir with the VEGA instrument (Sect. 2) and the determination of its limb-darkened angular diameter. To fix the star's position in the HR diagram, we compute the star's bolometric flux (Sect. 3) and derive its fundamental parameters (Sect. 4). We then use a stellar evolution code to determine the mass and the age of the target and a non-adiabatic pulsation code (Balmforth et al. 2001) to predict its pulsation properties (Sect. 5). Finally we compare our results with those published in the literature and conclude (Sect. 6).

\section{Interferometric observations and data processing}

\subsection{CHARA/VEGA observations}

We used the visible VEGA spectrograph (Mourard et al. 2009) installed at the combined focus of the CHARA array which hosts six one-meter telescopes and allows a maximum baseline of about $330 \mathrm{~m}$ to be reached (Ten Brummelaar et al. 2010). Combined with the visible wavelengths of VEGA, an angular resolution of $\lambda /(2 B) \sim 0.2$ mas can be achieved, which is particularly effective in order to resolve the Ap stars. We always combine three telescopes of the array to probe long baselines (i.e. longer than $200 \mathrm{~m}$ ) and record the spectrally dispersed fringes around $700 \mathrm{~nm}$ with a spectral resolution of 6000 . This mode has a limiting correlated magnitude of $m_{\mathrm{V}} \sim 6.5$ in medium seeing conditions, which is well adapted to our target. We used the $K$-band CLIMB beam combiner in parallel with VEGA to ensure the fringe coherencing (Sturmann et al. 2010). We followed a sequence of calibrator-target-calibrator, with 40 blocks of 2500 short exposures (of $10 \mathrm{~ms}$ ) per star. Swapping from one star to another every 20 min ensures that the instrumental transfer function is stable enough. We selected the calibrators with the SearchCal tool (Bonneau et al. 2006) ${ }^{1}$ : HD 109704, HD 127167 ,

\footnotetext{
1 wWW. jmmc.fr/searchcal
}

Table 1. $V$ magnitude and uniform-disk angular diameters of the calibrators in the $R$ band.

\begin{tabular}{ccc}
\hline \hline HD number & $m_{V}$ & $\theta_{\mathrm{UD}}(R)(\mathrm{mas})$ \\
\hline 109704 & 5.87 & $0.218 \pm 0.016$ \\
127167 & 5.96 & $0.258 \pm 0.018$ \\
129956 & 5.67 & $0.219 \pm 0.016$ \\
130557 & 6.13 & $0.159 \pm 0.011$ \\
\hline
\end{tabular}

HD 129956, and HD 130557. We checked their uniform-disk angular diameters with the getCal database ${ }^{2}$ and used the uniformdisk angular diameters provided by the JSDC catalog (Lafrasse et al. 2010) in the $R$ band (Table 1). The observation log is given in Table 1, which includes the date and the time of the observations, the telescope pair, the projected baseline length $B_{\mathrm{p}}$ and its orientation PA, the central wavelength $\lambda_{0}$ of the spectral range used for computing the squared visibility $V^{2}$ and the errors $\sigma$ on $V^{2}$ (see Sect. 2.2), the Fried parameter $r_{0}$, and the HD number of the calibrators. Except for the shortest baseline, for which the $\mathrm{PA}$ is $-83^{\circ}$, all the baseline orientations are close to each other $\left(\mathrm{PA}=108^{\circ} \pm 6^{\circ}\right)$ and we will not be able to determine any angular diameter variation with respect to the baseline orientation.

\subsection{VEGA data processing}

The interferometric data were reduced using the standard VEGA reduction pipeline described in Mourard et al. (2009) which computes the squared visibility in wide spectral bands based on a spectral density analysis. For each night, we first checked that the instrumental transfer function was stable over the night by computing this transfer function for all the calibrators of all the programs of the night as explained in Mourard et al. (2012). Bad sequences due to poor seeing conditions or instrumental instabilities noted in the journal of the night were rejected.

For each data sequence (calibrator-target-calibrator) we computed the raw squared visibility for each block of 1000 individual frames and for different spectral bands of $15 \mathrm{~nm}$ in the continuum (whose central wavelengths $\lambda_{0}$ are given in Table 2). Following the method used for 10 Aql (Perraut et al. 2013), we only considered the squared visibilities obtained for a signalto-noise ratio higher than 4 . Using the known angular diameter of the calibrators, we calibrated the target squared visibilities $V^{2}$ and estimated a weighted mean calibrated squared visibility and the corresponding errors (Table 2): $\sigma_{\text {stat }}$ corresponds to the statistical dispersion over the block's measurements and $\sigma_{\text {syst }}$ accounts for the uncertainty on the calibrator diameter. We obtained 15 visibility points, each affected by a global er$\operatorname{ror} \sigma=\sqrt{\sigma_{\text {stat }}^{2}+\sigma_{\text {syst }}^{2}}$. Even for the longest baselines (i.e. more than $300 \mathrm{~m}$ ) these global errors remain dominated by the measurement dispersion and not by the accuracy on the calibrators' diameter, mainly because for such long baselines the observations on the calibrators that are faint are difficult and the dispersion on visibility measurement can be large.

\subsection{Deriving uniform-disk and limb-darkened angular diameters}

Figure 1 presents all the calibrated squared visibilities as a function of the spatial frequency $\left(B_{p} / \lambda\right)$. Since all visibility measurements are in the first lobe of the visibility curve, they only provide the angular size of the target. In particular our visibility

2 http://nexsci.caltech.edu/software/getCal/ 
Table 2. Log of the observations.

\begin{tabular}{|c|c|c|c|c|c|c|c|c|c|c|c|}
\hline \multirow[t]{2}{*}{ Date } & \multirow{2}{*}{$\begin{array}{l}\text { UT } \\
\text { (h) }\end{array}$} & \multirow{2}{*}{$\begin{array}{l}\text { HA } \\
\text { (h) }\end{array}$} & \multicolumn{3}{|c|}{ Baselines } & \multirow{2}{*}{$\begin{array}{c}\lambda_{0} \\
(\mathrm{~nm})\end{array}$} & \multirow[t]{2}{*}{$V^{2}$} & \multirow[t]{2}{*}{$\sigma_{\text {stat }}$} & \multirow[t]{2}{*}{$\sigma_{\text {syst }}$} & \multirow{2}{*}{$\begin{array}{c}r_{0} \\
(\mathrm{~cm})\end{array}$} & \multirow{2}{*}{$\begin{array}{c}\text { Cal } \\
\text { (HD) }\end{array}$} \\
\hline & & & Telescopes & $B_{\mathrm{p}}(\mathrm{m})$ & $\mathrm{PA}\left({ }^{\circ}\right)$ & & & & & & \\
\hline \multirow[t]{2}{*}{ 2012-Apr-17 } & 7.7 & 0 & E2W2 & 152 & -113 & 707.5 & 0.759 & 0.043 & 0.013 & $\sim 7$ & 130557,129956 \\
\hline & 7.7 & 0 & E1W2 & 214 & -114 & 707.5 & 0.525 & 0.067 & 0.022 & $\sim 7$ & 130557 \\
\hline \multirow[t]{3}{*}{ 2013-May-25 } & 5.5 & 0.3 & E2W1 & 248 & -102 & 707.5 & 0.481 & 0.018 & 0.031 & $\sim 13$ & 127167,129956 \\
\hline & & & E1W1 & 307 & -105 & 707.5 & 0.234 & 0.033 & 0.021 & & 129956 \\
\hline & & & E2W1 & 248 & -102 & 752.5 & 0.486 & 0.031 & 0.023 & & 129956 \\
\hline \multirow[t]{6}{*}{ 2014-May-03 } & 5.2 & -1.4 & $\overline{\mathrm{W} 1 \mathrm{~W} 2}$ & 93 & -83 & 707.5 & 0.913 & 0.044 & 0.005 & $\sim 10$ & 109704,129956 \\
\hline & & & E1W2 & 221 & -112 & 707.5 & 0.550 & 0.078 & 0.021 & & 109704,129956 \\
\hline & & & E1W1 & 306 & -104 & 707.5 & 0.282 & 0.034 & 0.024 & & 109704 \\
\hline & & & W1W2 & 93 & -83 & 727.5 & 0.935 & 0.073 & 0.007 & $\sim 10$ & 109704 \\
\hline & & & E1W2 & 221 & -112 & 727.5 & 0.452 & 0.112 & 0.017 & & 109704 \\
\hline & & & E1W1 & 306 & -104 & 727.5 & 0.333 & 0.083 & 0.026 & & 109704 \\
\hline \multirow[t]{2}{*}{ 2014-Мay-03 } & 6.2 & -0.4 & E1W2 & 219 & -113 & 707.5 & 0.444 & 0.034 & 0.016 & & 130557,129956 \\
\hline & & & E1W1 & 313 & -104 & 707.5 & 0.258 & 0.042 & 0.022 & & 129956 \\
\hline \multirow[t]{2}{*}{ 2014-May-04 } & 5.0 & -1.6 & E1W2 & 220 & -112 & 709 & 0.486 & 0.059 & 0.020 & $\sim 10$ & 109704 \\
\hline & & & E1W1 & 302 & -104 & 709 & 0.241 & 0.063 & 0.019 & & 109704,129956 \\
\hline
\end{tabular}

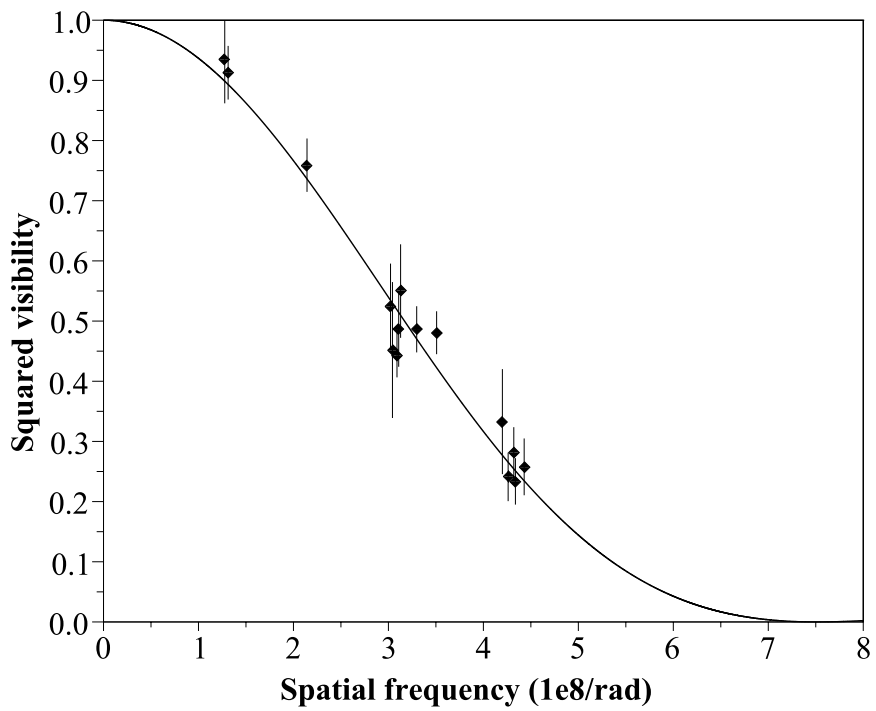

Fig. 1. Squared visibility versus spatial frequency for 78 Vir obtained with the VEGA observations (diamonds). The solid line represents the uniform-disk best model provided by LITpro.

points do not allow us to resolve the surface spots of this peculiar star, neither spatially (the used baselines are too small) nor spectrally (the spectral band along which we compute visibilities is too wide). Using the model fitting tool LITpro $^{3}$ we fitted all the visibility measurements versus spatial frequency, leading to a uniform-disk angular diameter $\theta_{\text {UD }}$ of $0.334 \pm 0.006$ mas for 78 Vir with a $\chi_{r}^{2}$ of 0.73 .

We used the linear limb-darkening coefficient $u(R)$ in the $R$ band provided by the Claret tables to derive the limb-darkened angular diameter $\theta_{\mathrm{LD}}$ of $78 \mathrm{Vir}$ through the formula:

$\theta_{\mathrm{LD}}=\theta_{\mathrm{UD}} \sqrt{\frac{1-\frac{u(R)}{3}}{1-7 \frac{u(R)}{15}}}$.

As detailed in Claret \& Bloemen (2011), $u(R)$ is obtained by a least-square method of adjustment of the radial intensity distribution on the stellar disk using a stellar atmosphere model characterized by the effective temperature $\left(T_{\text {eff }}\right)$, the gravity $(\log g)$, the metallicity $(Z)$, and the microturbulent velocity $(x i)$. For a

\footnotetext{
www.jmmc.fr/litpro_page.htm
}

solar metallicity and a null microturbulent velocity, we computed the coefficients $u(R)$ and the corresponding limb-darkened diameters when the effective temperature spans from $8750 \mathrm{~K}$ to $9250 \mathrm{~K}$ and $\log g$ from 4 to 5 . When scanning the gravity range, the limb-darkened diameter only varies at the level of $10^{-4}$ mas; instead, when scanning the temperature range, differences smaller than $10^{-3}$ mas are noticed. We also tested different metallicities from -5 to +1 : over this range the limbdarkened diameter only varies by $10^{-3}$ mas. These variations are 5-7 times smaller than our error bar on the uniform-disk diameter. By considering the average limb-darkening coefficient on our parameter space, $u(R)=0.428$, we obtained a limb-darkened angular diameter of $\theta_{\mathrm{LD}}=0.346 \pm 0.006$ mas for $78 \mathrm{Vir}$, hence an accuracy better than $2 \%$.

\section{Computation of the bolometric flux}

Next we compute the bolometric flux for 78 Vir following the same procedure as in Perraut et al. (2013). The apparent flux distribution was obtained by combining photometric and spectroscopic data available in the literature, in particular:

- For wavelengths in the range $1150 \AA<\lambda<3320 \AA$, we combined 25 rebinned spectra from the Sky Survey Telescope obtained at the IUE Newly Extracted Spectra (INES) data archive ${ }^{4}$. Based on the quality flag listed in the IUE spectra (Garhart et al. 1997) we removed all bad pixels from the data, and we also removed the points with negative flux.

- For wavelengths in the range $3320 \AA<\lambda<3904 \AA$ and $6800 \AA<\lambda<7850 \AA$, we combined the spectrophotometric data from Kharitonov et al. (1988), Adelman et al. (1989), and Glushneva et al. (1998).

- For wavelengths in the range $3904 \AA<\lambda<6800 \AA$, we combined two flux-calibrated ELODIE spectra extracted from the ELODIE.3.1 library $^{5}$ (Prugniel \& Soubiran 2001; Prugniel et al. 2007).

- At the longest wavelengths, we collected the photometric data from the 2MASS All Sky Catalog of point sources 6 (Cutri et al. 2003). Filter responses and zero points were taken from Cohen et al. (2003).

\footnotetext{
4 http://sdc.laeff.inta.es/cgi-ines/IUEdbsMY

5 http://www.obs.u-bordeaux1.fr/m2a/soubiran/elodie_ library.html

6 http://irsa.ipac.caltech.edu/applications/Gator/
} 


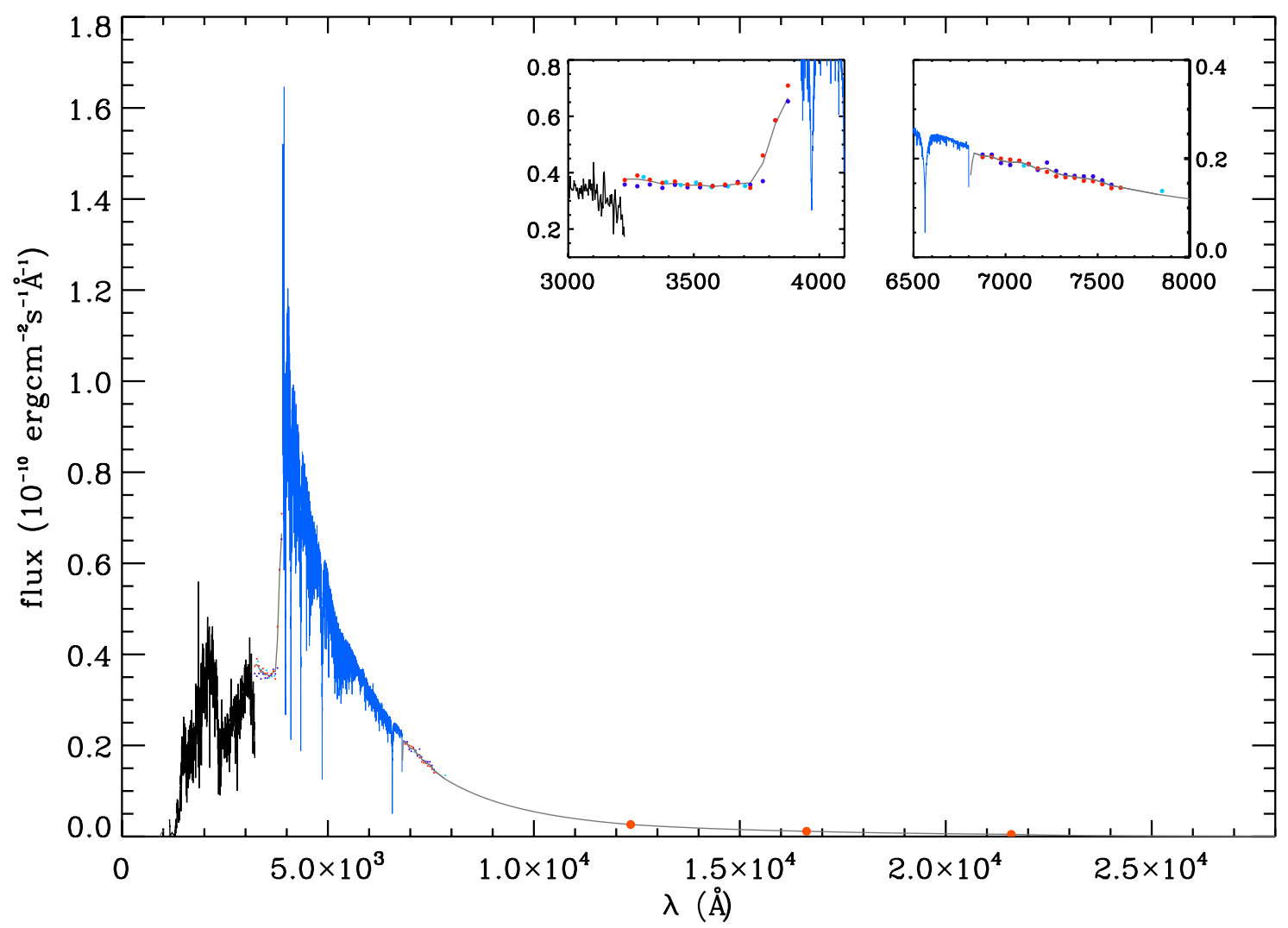

Fig. 2. Spectral energy distribution obtained for 78 Vir. The IUE spectrum is shown in black at the lower wavelengths, the ELODIE spectrum is shown in blue, the interpolations are shown in gray and the 2MASS data are shown by orange circles. Spectrophotometric data are shown by blue (Kharitonov et al. 1988), cyan (Adelman et al. 1989), and red (Glushneva et al. 1998) dots. Insets at the top are zooms for two different spectral ranges. (See online edition for a color version.)

The spectral energy distribution derived from these data is shown in Fig. 2, where we interpolated linearly (in logarithmic scale) between the photometric and the spectrophotometric data points, and at both extremes of the spectral distribution, considering zero flux at $912 \AA$ and at $1.6 \times 10^{6} \AA$. In the case of the spectrophotometric observations we interpolated the data points from each catalog separately and then took the average curve. By integrating the spectral energy distribution we obtained the bolometric flux, $f_{\text {bol }}=2.73 \pm 0.20 \times 10^{-7} \mathrm{erg} / \mathrm{cm}^{2} / \mathrm{s}$. The uncertainties were estimated by considering the values quoted in the corresponding data sources, in wavelength regions where calibrated spectra were used, and a conservative value of $15 \%$ uncertainty for the remaining wavelengths where interpolations were made.

\section{Determination of the fundamental parameters}

\subsection{Linear radius}

We derived the radius of 78 Vir and its error through a Monte Carlo simulation,

$R \pm \delta R=\frac{\theta_{\mathrm{LD}}+\delta \theta_{\mathrm{LD}}}{9.305 \times\left(\pi_{\mathrm{P}}+\delta \pi_{\mathrm{P}}\right)}$

where $R$ stands for the stellar radius (in solar radius, $R_{\odot}$ ), $\theta_{\mathrm{LD}}$ for the limb-darkened angular diameter (in mas), and $\pi_{\mathrm{P}}$ for the parallax (in seconds of arc). By taking $\pi_{\mathrm{P}}=17.65 \pm 0.20$ mas (van Leeuwen 2007) we obtained $R=2.11 \pm 0.04 R_{\odot}$, hence an accuracy of $1.9 \%$ on the radius determination.

\subsection{Luminosity and effective temperature}

We used the parallax $\pi_{\mathrm{P}}$ and the bolometric flux $f_{\text {bol }}$ to derive the luminosity through a Monte Carlo method,

$L=4 \pi f_{\mathrm{bol}} C^{2} / \pi_{\mathrm{p}}^{2}$,

where $C$ is the conversion from parsec to $\mathrm{cm}\left(3.086 \times 10^{18}\right)$, and $\pi_{\mathrm{p}}$ the parallax in seconds. We found $L=27.3 L_{\odot}$. The error bar is strongly dominated by that of the bolometric flux. We thus fix $L=27 \pm 2 L_{\odot}$.

We used the measured angular diameter and bolometric flux to estimate the effective temperature through the equation

$\sigma T_{\mathrm{eff}}^{4}=4 f_{\mathrm{bol}} / \theta_{\mathrm{LD}}^{2}$

where $\sigma$ stands for the Stefan-Boltzmann constant $(5.67 \times$ $\left.10^{-5} \mathrm{erg} \mathrm{cm}^{-2} \mathrm{~s}^{-1} \mathrm{~K}^{-4}\right)$. We determined an effective temperature of $9100 \pm 190 \mathrm{~K}$.

\section{Stellar and pulsation properties}

\subsection{Position in the Hertzsprung-Russell diagram}

We used the fundamental parameters derived in Sect. 4 to put 78 Vir in the Hertzsprung-Russell (HR) diagram. To derive the mass and the age of our target, we used the isochrone tool CMD $2.5^{7}$ with $Z=0.019, Y=0.28242,[\mathrm{M} / \mathrm{H}]=0.119$ (Fig. 3). Thanks to our accurate determination of the radius, we obtained a mass of $2.16 \pm 0.04 M_{\odot}$ and an age of $435 \pm 55 \mathrm{Myr}$. All the fundamental parameters are gathered in Table 3 .

7 http://stev.oapd.inaf.it/cmd 
Table 3. Fundamental parameters of 78 Vir.

\begin{tabular}{cccc}
\hline \hline Parameters & Monier (1992) & Khalack \& Wade (2006) & This work \\
\hline$\theta_{\mathrm{LD}}(\mathrm{mas})$ & $0.343 \pm 0.024$ & - & $0.346 \pm 0.006$ \\
$d(\mathrm{pc})$ & $48 \pm 15$ & $56.2 \pm 2.5^{a}$ & $56.7 \pm 0.6^{b}$ \\
$R\left(R_{\odot}\right)$ & $1.77 \pm 0.68$ & $2.06 \pm 0.17$ & $2.11 \pm 0.04$ \\
$f_{\text {bol }}\left(10^{-7} \mathrm{erg} / \mathrm{cm}^{2} / \mathrm{s}\right)$ & $2.7 \pm 0.3$ & - & $2.73 \pm 0.20$ \\
$L\left(L_{\odot}\right)$ & - & $27.3 \pm 2.5$ & $27 \pm 2$ \\
$T_{\text {eff }}(\mathrm{K})$ & $9200 \pm 290$ & from Monier $(1992)$ & $9100 \pm 190$ \\
$M\left(M_{\odot}\right)$ & - & $2.18 \pm 0.06$ & $2.16 \pm 0.04$ \\
age $(\mathrm{Myr})$ & - & $300+70 /-130$ & $435 \pm 55$ \\
\hline
\end{tabular}

Notes. ${ }^{(a)}$ From the HIPPARCos and Tycho catalogs (ESA SP-1200; Noordwijk: ESA); ${ }^{(b)}$ from the parallax of van Leeuwen (2007).

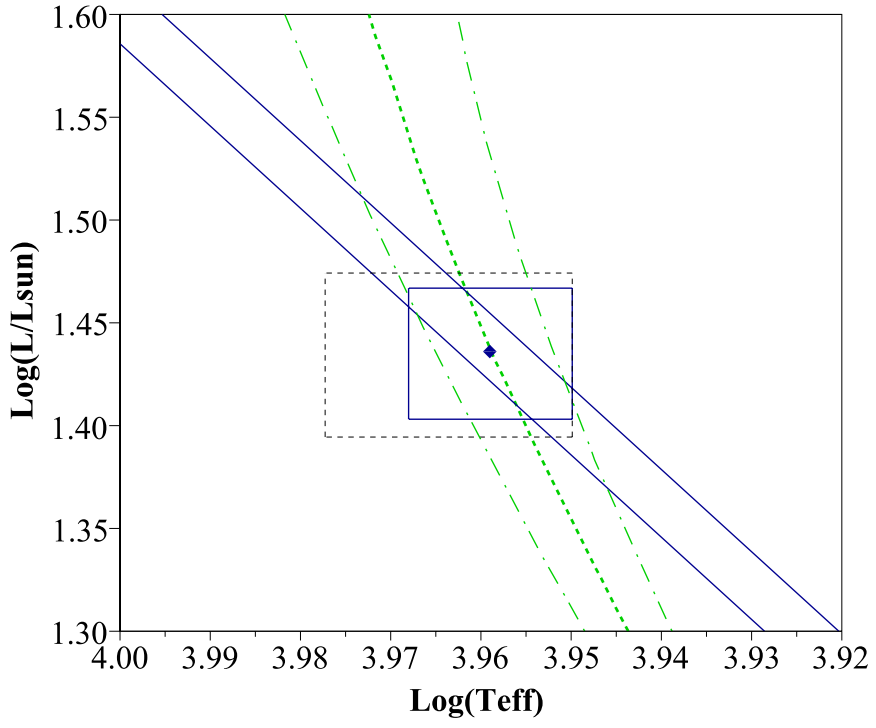

Fig. 3. Position of 78 Vir in the Hertzsprung-Russell diagram. The constraints on the fundamental parameters are indicated by the $1 \sigma$-error box $\left(\log \left(T_{\text {eff }}\right), \log \left(L / L_{\odot}\right)\right)$ and the diagonal lines (radius). The blue box in solid lines corresponds to our work and the black box in dashed lines to the parameters derived by Khalack \& Wade (2006). Isochrones are overplotted in green lines for $Z=0.019, Y=0.28242,[\mathrm{M} / \mathrm{H}]=0.119$ and ages of $435 \mathrm{Myr}$ (best fit - dashed line), $380 \mathrm{Myr}$, and $490 \mathrm{Myr}$ (dash-dotted lines). (See online edition for a color version.)

\subsection{Non-adiabatic pulsation analysis}

The effective temperature and luminosity derived from our work place 78 Vir within the roAp stars' theoretical instability strip (Cunha 2002, the theoretical red and blue edges derived by the author are reproduced in our Fig. 4). This makes 78 Vir an important target for extending the tests on the roAp stars' excitation mechanism towards the theoretical blue edge. With this in mind, we have used the fundamental parameters determined in this work to perform a linear, non-adiabatic stability analysis for 78 Vir. The analysis follows closely those presented by Balmforth et al. (2001) and Cunha et al. (2013). The equilibrium models are composed of two different regions: the equatorial region, where envelope convection proceeds normally, and the polar region, where envelope convection is assumed to be suppressed by the magnetic field. In practice, two spherically symmetric models are built, each with the characteristics of one of the regions. The models are then matched below the convectively unstable layers to guarantee that they have the same internal structure. The computation of the models requires the input of the stellar mass, luminosity, effective temperature, and internal chemical composition. In addition, it requires the definition

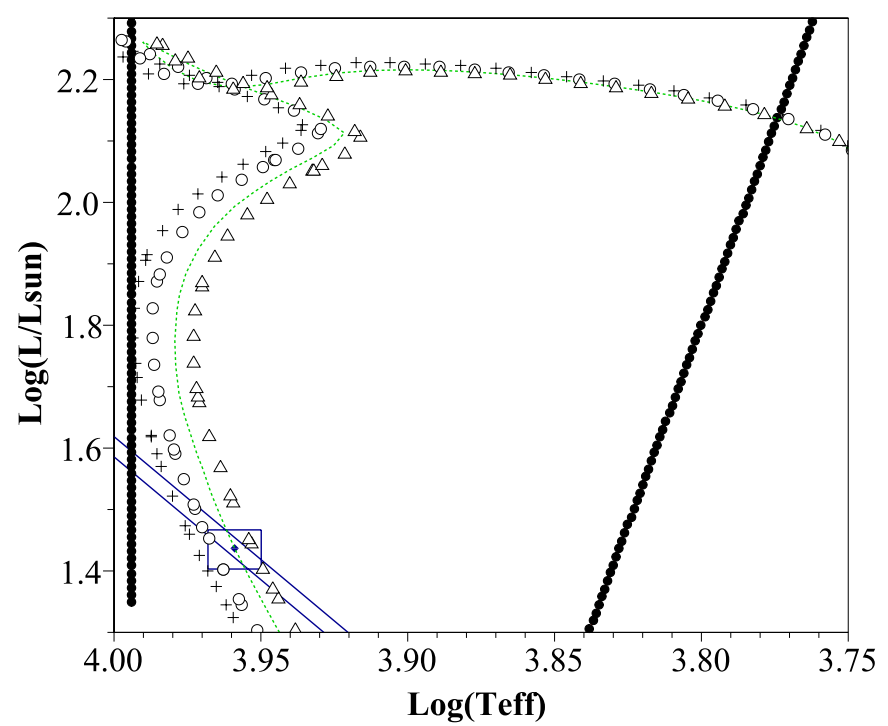

Fig. 4. Position of 78 Vir in the Hertzsprung-Russell diagram. Our constraints on the fundamental parameters are indicated by the blue $1 \sigma$-error box $\left(\log \left(T_{\text {eff }}\right), \log \left(L / L_{\odot}\right)\right)$ and the blue diagonal lines (radius). For a fixed age of $435 \mathrm{Myr}$, isochrones are overplotted for $Z=0.014$ (plus), $Z=0.016$ (open circles), $Z=0.019$ (best fit - dotted green line), and $Z=0.022$ (open triangles). Filled symbols mark the blue and red edges of the instability strip. (See online edition for a color version.)

of the minimum optical depth in the atmosphere and of the surface helium abundance. The latter may differ from the helium abundance in the interior owing to helium settling. The stability analysis is carried out separately in the two regions, the results corresponding to two limit cases: the case of a star in which envelope convection is fully suppressed by the magnetic field and the case of a star in which the magnetic field does not affect envelope convection at all. These results can then be combined to obtain the growth rates in a model with convection suppressed only in some angular region around the magnetic pole (hereafter called the composite model). In the pulsation analysis it is possible to choose between two mechanical outer boundary conditions: one that imposes the full reflection of the waves and one that allows waves with frequency above the acoustic cutoff frequency to propagate. The latter condition is derived by matching the computed solutions with the analytical solutions valid for a plane-parallel isothermal atmosphere. We refer the reader to the works mentioned above for a more detailed description of the models and corresponding physical assumptions.

To account for the main model uncertainties, we have considered four different cases. In all of them we have adopted a homogenous helium abundance for the equatorial regions, in 
Table 4. Modeling parameters for the cases illustrated in Fig. 5, all computed with $M=2.16 M_{\odot}, T_{\text {eff }}=9100 \mathrm{~K}, L / L_{\odot}=27$.

\begin{tabular}{cccc}
\hline \hline Model & Polar $Y_{\text {surf }}$ & $\tau_{\min }$ & $\begin{array}{c}\text { Boundary } \\
\text { condition }\end{array}$ \\
\hline $\mathrm{A}$ & 0.01 & $1.5 \times 10^{-4}$ & Reflective \\
$\mathrm{B}$ & 0.01 & $3.5 \times 10^{-4}$ & Reflective \\
$\mathrm{C}$ & 0.01 & $1.5 \times 10^{-4}$ & Transmissive \\
$\mathrm{D}$ & 0.1 & $1.5 \times 10^{-4}$ & Reflective \\
\hline
\end{tabular}

accordance with the results of Théado et al. (2005). In the first case (hereafter case A), the equilibrium model is characterized by a minimum optical depth of $\tau_{\min }=1.5 \times 10^{-4}$ and a surface helium abundance in the polar region of $Y_{\text {surf }}=0.01$. Moreover, in the pulsation analysis for this case we apply a fully reflective boundary condition at the surface. The other three cases are obtained by changing these properties, one at the time (keeping the other properties equal to case A). The details are summarized in Table 4.

The results of our non-adiabatic calculations are shown in Fig. 5, where we plot the relative growth rates, $\eta / \omega$, as a function of the cyclic frequency, $v$. Here $\eta$ and $\omega$ are the imaginary and real parts of the angular eigenfrequencies, respectively. All highovertone pulsations are predicted to have negative growth rates in models with the properties of the equatorial region (open symbols). This is in agreement with earlier results found for models of other roAp stars (e.g. Cunha et al. 2013). It implies that regions in which envelope convection is not suppressed contribute to stabilizing the oscillations. In contrast, the results for the polar models (filled symbols) all show positive growth rates for a range of high overtones.

To make an inference about the stability of the oscillations in the composite model we must compare the absolute values of the growth rates in the polar and equatorial regions. Following Balmforth et al. (2001, see their Eq. (15) and their Fig. 4), the analysis predicts that high overtone dipolar pulsations aligned with the magnetic field axis $(l=1, m=0)$ would be excited in 78 Vir if the envelope convection is suppressed in an angular region (hereafter spot) larger than about $40^{\circ}$ around the magnetic pole. For the excitation of spherically symmetric modes $(l=0)$ the spot would have to be larger than about $60^{\circ}$, while the excitation of $l=1, m= \pm 1$ modes would require a spot with an extension of at least $70^{\circ}$.

The composite model proposed by Balmforth et al. (2001), hence also the size of the spots discussed above, assumes that the pulsation axis is aligned with the magnetic axis. This is the case when the magnetic field effect dominates over the effect of rotation on the oscillations. Given the equatorial rotational velocity of $78 \operatorname{Vir}\left(V_{e}=29 \pm 4 \mathrm{~km} \mathrm{~s}^{-1}\right.$; Khalack \& Wade 2006), one may expect the rotational effect on the pulsations to be nonnegligible, possibly leading to a non-alignment between the pulsation axis and the magnetic field axis (Bigot \& Dziembowski 2002; Bigot \& Kurtz 2011). Still, the angle between the magnetic and rotation axes is quite significant in $78 \mathrm{Vir}$ (with an obliquity $\beta=124^{\circ} \pm 5^{\circ}$, derived by Khalack \& Wade 2006), which, according to the results of Bigot \& Dziembowski (2002), favors a quasi-alignment of one of the dipolar modes with the magnetic field, making the $40^{\circ}$ minimum requirement for the spot size a reasonable expectation. In the worst scenario of a significant non-alignment between the pulsation and magnetic axes, the minimum size of the spot required for the excitation of pulsations in 78 Vir may be larger than $40^{\circ}$, but still not larger than about $60^{\circ}$, since the condition on the excitation of

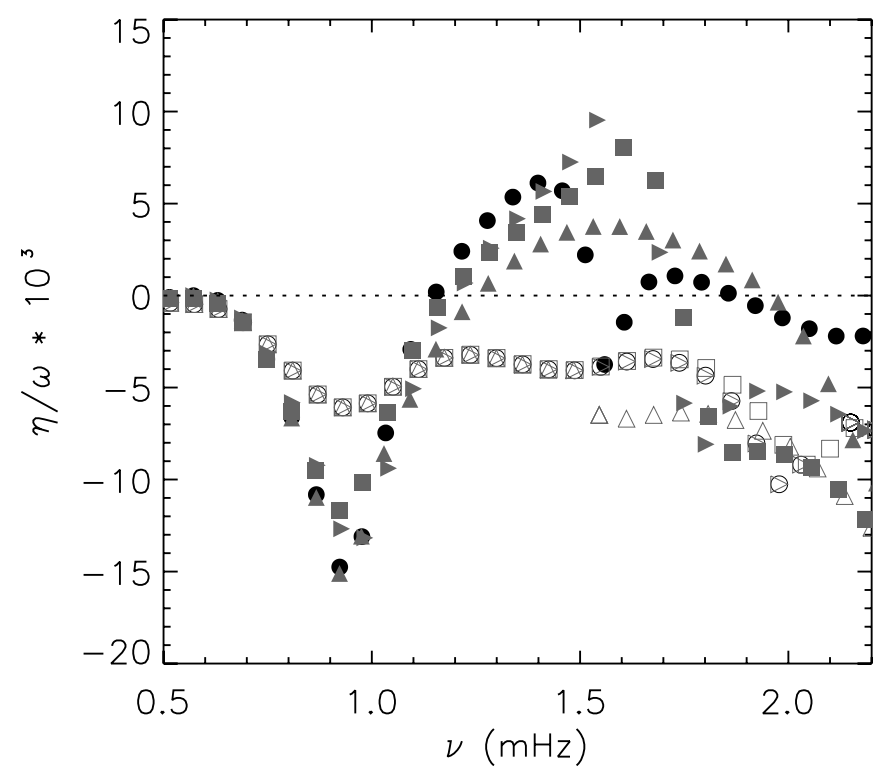

Fig. 5. Relative growth rates for the four cases considered. Open symbols show the results for the equatorial models (with envelope convection) and filled symbols show the results for the polar models (without envelope convection): case A (circles); case B (squares); case C (upwards triangles); case D (right facing triangles). The horizontal dotted line marks where $\eta / \omega=0$. See Table 4 for a description of the four cases.

the $l=0$ modes is unchanged. Whatever the case, if modes are indeed excited in 78 Vir they are expected to be found within the frequency interval $\sim 1.2-1.9 \mathrm{mHz}$.

Finally, we would like to note that the convectively unstable region in 78 Vir is rather shallow, making the suppression of convection in this star relatively easy when compared to the known roAp stars (Cunha 2002). Given the strength of the magnetic field observed in $78 \mathrm{Vir}$, we can thus conclude that the case for the driving of high-overtone pulsations in 78 Vir is very strong.

\section{Discussion and conclusion}

Thanks to interferometric measurements in the visible range we managed to clearly resolve 78 Vir and derive an angular diameter that is as independent as possible of atmosphere models. We obtained a limb-darkened angular diameter of $\theta_{\mathrm{LD}}=$ $0.346 \pm 0.006$ mas, which is close to that derived by Monier (1992) from infrared measurements, the bolometric flux, and predictions of atmosphere models $\left(\theta_{\text {mod }}=0.343 \pm 0.024\right.$ mas $)$. Our radius differs from that of Monier (1992) only because they considered a different distance (see Table 3 ). Our radius is in good agreement with the value determined by Khalack \& Wade (2006). We obtained similar bolometric flux and effective temperature to Monier (1992) and our effective temperature and luminosity are consistent with the values derived by Khalack \& Wade (2006) as depicted in Fig. 3. In addition, Monier (1992) noticed in his paper that previous effective temperature determinations pointed towards much higher values, i.e. above $9500 \mathrm{~K}$ and up to $10700 \mathrm{~K}$, which is strongly incompatible with our angular diameter measurement. As an example, an effective temperature of $10000 \mathrm{~K}$ leads to a radius of $1.74 R_{\odot}$ and an angular diameter of 0.286 mas, which is completely ruled out by our measurements.

Our mass is in close agreement with that of Khalack \& Wade (2006), but the age is marginally consistent with their 
determination even if we considered a similar $Z$. Our age is in agreement with the value of $500 \pm 100 \mathrm{Myr}$ of King et al. (2003), but the authors considered a much higher effective temperature (9710 K) than we did. King et al. (2003) also flag 78 Vir as an uncertain member of the Ursa Major stream. Based on the metallicity $[\mathrm{Fe} / \mathrm{H}]$ whose $\log$ spans from -0.13 to +1.58 with respect to the solar metallicity (Cayrel de Strobel et al. 1997), it is not clear whether this star has a solar metallicity, hence the uncertainties of the mass and the age can be large. Figure 4 presents isochrones for a given age (435 Myr) and different values of $Z$. Thanks to our constraints on $\log \left(T_{\text {eff }}\right), \log \left(L / L_{\odot}\right)$, and also on $R$ we derived a mass of $2.15 M_{\odot}$ for $Z=0.016$ and $Z=0.022$, which is in perfect agreement with our determination for a solar metallicity. A mass of $2.07 M_{\odot}$ for $Z=0.014$ can be derived while only considering the constraints on $\log \left(T_{\text {eff }}\right)$ and $\log \left(L / L_{\odot}\right)$, but the corresponding isochrone does not fit our constraints on the radius. As pointed out by Creevey et al. (2007) an accurate determination of the radius is thus a powerful means of deriving accurate masses.

To our knowledge there are no studies of the pulsation properties of 78 Vir published in the literature, nor any reported attempts to observe rapid pulsations in this star. However, 78 Vir appears to be a good candidate to extend the tests on the pulsation excitation mechanism for roAp stars towards the blue edge of the instability strip. Given its asteroseismic potential, data for this star have recently been acquired with the SONG telescope network (Antoci et al., in prep.).

Acknowledgements. The authors want to warmly thank R. Monier for the fruitful discussions. VEGA is supported by French programs for stellar physics and high angular resolution PNPS and ASHRA, by the Observatoire de la Côte d'Azur and the Laboratoire Lagrange. The CHARA Array is operated with support from the National Science Foundation through grant AST-0908253, the W.M. Keck Foundation, the NASA Exoplanet Science Institute, and from Georgia State University. This work has been supported by a grant from LabEx OSUG@2020 (Investissements d'avenir - ANR10LABX56) and made use of funds from the ERC through the project FP7-SPACE-2012-312844. MSC is supported by FCT through the Investigador FCT contract of reference IF/00894/2012 and POPH/FSE (EC) by FEDER funding through the program COMPETE. Funds for this work were provided also by the EC, under FP7, through the project FP7-SPACE-2012-31284. I.B. acknowledges financial support from the FCT through the grant SFRH/BPD/87857/2012. This research has made use of the SearchCal and LITpro services of the Jean-Marie Mariotti Center, and of CDS Astronomical Databases SIMBAD and VIZIER.

\section{References}

Adelman, S. J., Pyper, D. M., Shore, S. N., White, R. E., \& Warren, Jr., W. H. 1989, A\&AS, 81, 221

Alentiev, D., Kochukhov, O., Ryabchikova, T., et al. 2012, MNRAS, 421, L82
Babcock, H. W. 1947, ApJ, 105, 105

Balmforth, N. J., Cunha, M. S., Dolez, N., Gough, D. O., \& Vauclair, S. 2001, MNRAS, 323, 362

Bigot, L., \& Dziembowski, W. A. 2002, A\&A, 391, 235

Bigot, L., \& Kurtz, D. W. 2011, A\&A, 536, A73

Blackwell, D. E., \& Shallis, M. J. 1977, MNRAS, 180, 177

Bonneau, D., Clausse, J.-M., Delfosse, X., et al. 2006, A\&A, 456, 789

Cayrel de Strobel, G., Soubiran, C., Friel, E. D., Ralite, N., \& Francois, P. 1997, A\&AS, 124, 299

Claret, A., \& Bloemen, S. 2011, VizieR Online Data Catalog: J/A\&A/529/A75

Cohen, M., Wheaton, W. A., \& Megeath, S. T. 2003, AJ, 126, 1090

Cowley, A., Cowley, C., Jaschek, M., \& Jaschek, C. 1969, AJ, 74, 375

Creevey, O. L., Monteiro, M. J. P. F. G., Metcalfe, T. S., et al. 2007, ApJ, 659,616

Cunha, M. S. 2002, MNRAS, 333, 47

Cunha, M. S., Fernandes, J. M. M. B., \& Monteiro, M. J. P. F. G. 2003, MNRAS, 343,831

Cunha, M. S., Aerts, C., Christensen-Dalsgaard, J., et al. 2007, A\&ARv, 14, 217

Cunha, M. S., Alentiev, D., Brandão, I. M., \& Perraut, K. 2013, MNRAS, 436, 1639

Cutri, R. M., Skrutskie, M. F., van Dyk, S., et al. 2003, VizieR Online Data Catalog: II/246

Garhart, M. P., Smith, M. A., Turnrose, B. E., Levay, K. L., \& Thompson, R. W. 1997, IUE NASA Newsletter, 57, 1

Glushneva, I., Doroshenko, V., Fetisova, T., et al. 1998, VizieR Online Data Catalog: III/208

Jugaku, J., \& Sargent, W. L. W. 1968, ApJ, 151, 259

Khalack, V. R., \& Wade, G. A. 2006, A\&A, 450, 1157

Kharitonov, A. V., Tereshchenko, V. M., \& Knyazeva, L. N. 1988, VizieR Online Data Catalog: III/202

King, J. R., Villarreal, A. R., Soderblom, D. R., Gulliver, A. F., \& Adelman, S. J. 2003, AJ, 125, 1980

Kochukhov, O., \& Wade, G. A. 2010, A\&A, 513, A13

Kochukhov, O., Drake, N. A., Piskunov, N., \& de la Reza, R. 2004, A\&A, 424,935

Kurtz, D. W., \& Martinez, P. 2000, Balt. Astron., 9, 253

Kurtz, D. W., Elkin, V. G., Cunha, M. S., et al. 2006, MNRAS, 372, 286

Lafrasse, S., Mella, G., Bonneau, D., et al. 2010, VizieR Online Data Catalog: II/300

Lüftinger, T., Kuschnig, R., Piskunov, N. E., \& Weiss, W. W. 2003, A\&A, 406, 1033

Lüftinger, T., Kochukhov, O., Ryabchikova, T., et al. 2010, A\&A, 509, A71

Michaud, G. 1970, ApJ, 160, 641

Monier, R. 1992, A\&A, 263, 175

Mourard, D., Clausse, J. M., Marcotto, A., et al. 2009, A\&A, 508, 1073

Mourard, D., Challouf, M., Ligi, R., et al. 2012, in SPIE Conf. Ser., 8445, 11

Perraut, K., Borgniet, S., Cunha, M., et al. 2013, A\&A, 559, A21

Prugniel, P., \& Soubiran, C. 2001, A\&A, 369, 1048

Prugniel, P., Soubiran, C., Koleva, M., \& Le Borgne, D. 2007, VizieR Online Data Catalog: III/251

Stift, M. J., \& Alecian, G. 2012, MNRAS, 425, 2715

Sturmann, J., Ten Brummelaar, T., Sturmann, L., \& McAlister, H. A. 2010, in SPIE Conf. Ser., 7734

Ten Brummelaar, T. A., McAlister, H. A., Ridgway, S. T., et al. 2010, in SPIE Conf. Ser., 7734

Théado, S., Vauclair, S., \& Cunha, M. S. 2005, A\&A, 443, 627

van Leeuwen, F. 2007, A\&A, 474, 653 